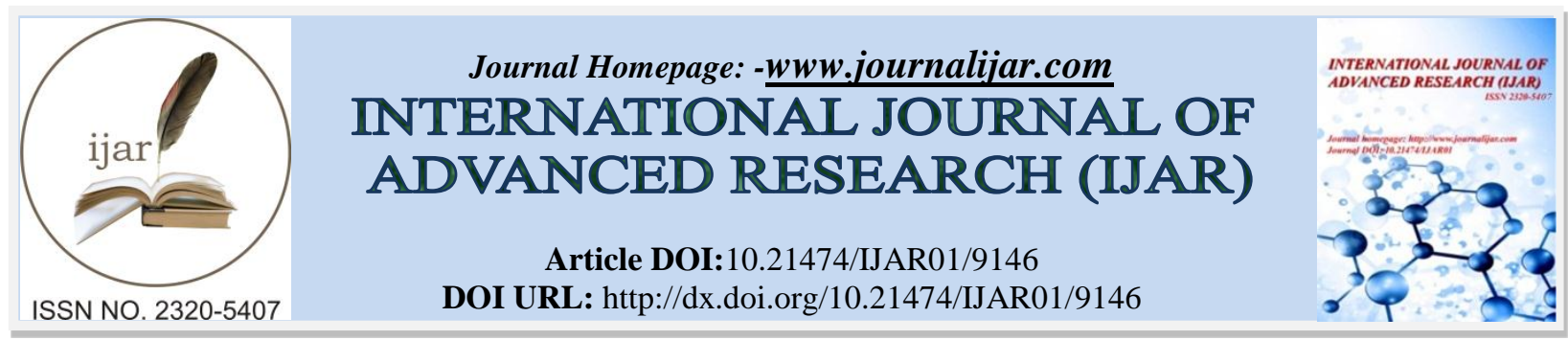

RESEARCH ARTICLE

\title{
MAGNETIC AND DIELECTRIC CHARACTERISATION OF PANI -COBALT FERRITE NANO COMPOSITE SYNTHESIZED VIA GREEN MEDIUM.
}

T.R.Smitha ${ }^{1}$, Thushara Bhadran ${ }^{2}$, Vaishali Shanker ${ }^{2}$ and K.H.Prema ${ }^{2}$.

1. Department of Chemistry, T.K.M.M.College, Nangiarkulangara, Alappuzha, Kerala, India.

2. Department of Chemistry, Sanatana Dharma College, Alappuzha, Kerala, India.

\section{Manuscript Info}

Manuscript History

Received: 24 March 2019

Final Accepted: 26 April 2019

Published: May 2019

Key words:-

Polyaniline, cobalt ferrite, composite, sol-gel.

\section{Abstract}

In recent years, the development of efficient green chemistry methods for synthesis of nano particles has become a major focus of researchers. In this work polyaniline (PANI) -cobalt ferrite nano composite is prepared using green solvent tamarind extract by oxidative chemical polymerization of aniline in presence of cobalt ferrite. Because of their unique electromagnetic properties, these composite nanoparticles have been increasingly employed in magnetic resonance imaging (MRI), targeted drug delivery and therapy. The structural characterization of the composite particles is done by XRD and IR and surface morphology by SEM. The magnetic properties of the samples prepared by varying the cobalt ferrite content in PANI are studied by VSM analysis and ac conductivity of the samples were calculated using the dielectric parameters measured using a dielectric cell.

Copy Right, IJAR, 2019,. All rights reserved.

\section{Introduction:-}

Organic-inorganic nano composites with an organized structure has been extensively studied because they combine the advantages of the inorganic materials (mechanical strength, electric and magnetic properties) and the organic polymers (flexibility, dielectric, ductility and processibility) (Subhash B.et.al, 2014; Veluru Jagadeesh Babu et al.2013) which are difficult to obtain from individual components. PANI-ferrite composites are usually investigated due to their unique magnetic and electrical characteristics (J. Stejskal et al., 2002; Jiaxing Huang, 2006). Under commercial point of view these polymer composites show promise for the production of various materials that can replace metals and semiconductors.

Polyaniline is the most widely studied conducting polymer because of its ease of synthesis, solubility, high chemical and thermal stability (E. E. Tanrıverdi, et al., 2011). Ferrites are usually non-conductive ceramic ferromagnetic compounds derived from iron oxides such as $\mathrm{Fe}_{2} \mathrm{O}_{3}$ or $\mathrm{Fe}_{3} \mathrm{O}_{4}$ as well as oxides of other metals, which have a wide range of technological applications (A. Tiwari,et al., 2007; .Y. Tan, et al., 2009; Yongli Li et al., 2015)

A number of groups have reported studies on the electrical conductivity and magnetic properties of composites of a variety of conducting polymers and ferrites. They found that the properties of the composites depend on several factors such as the type of filler, its concentration, size and strength of interaction between the filler molecules and the polymer macromolecules (G.D Prasanna, et al., 2014). In this work nano cobalt ferrite has been prepared by solgel method and cobalt ferrite reinforced PANI composite have been prepared by oxidative chemical polymerization

Corresponding Author:- K.H.Prema.

Address:-Department of Chemistry, Sanatana Dharma College, Alappuzha, Kerala, India. 
in green solvent tamarind extract other than the conventional solvents such as $\mathrm{HCl}, \mathrm{H}_{2} \mathrm{SO}_{4}$, etc. The nano cobalt ferrite particles were added in different concentration to the polymer matrix and variation in the magnetic properties and ac conductivity of the various samples are measured.

\section{Experimental}

The ferric nitrate and cobalt nitrate used for the synthesis of cobalt ferrite were purchased from Qualigens chemicals with $98 \%$ purity. The chemicals aniline and acetone used for the synthesis of polyaniline were purchased from Merck chemical company and ammonium peroxydisulphate (APS) from spectrochem and all are of high purity. Aniline is used after double distillation. Tamarind extract at $\mathrm{pH}=2$ is prepared in distilled water.

Synthesis of cobalt ferrite $(\mathrm{CF})$

Ferric nitrate and cobalt nitrate are dissolved in pure ethylene glycol at $40^{\circ} \mathrm{C}$ in the molar ratio $2: 1$ and the solution is heated to $60^{\circ} \mathrm{C}$. The temperature is kept constant till a wet gel of metal nitrate is obtained. The gel is dried at $100^{\circ} \mathrm{C}$, itself ignited to produce a fluffy product which is powdered into fine powder in presence of acetone medium and heated at $200^{\circ} \mathrm{C}$ in a muffle furnace to get fine powder.

Synthesis of pani-cobalt ferrite composite

0.4M Aniline monomer is dissolved in tamarind extract at $\mathrm{pH}$ 2. Pre-synthesized $\mathrm{CF}$ powder (in varying amount) is added slowly to the reaction medium with vigorous stirring in order to keep $\mathrm{CoFe}_{2} \mathrm{O}_{4}$ powder suspended in the solution. To this Ammonium Persulphate (APS) solution with aniline/persulphate ratio 1:1.25 is added drop wise with continuous stirring at $0-5^{\circ} \mathrm{C}$. The resulting precipitate is filtered under suction, washed with distilled water and then with acetone to dissolve any unreacted aniline. After washing, the precipitate is dried in an air oven at $50^{\circ} \mathrm{C}$ for 24 hours. The obtained composite is grinded into a fine powder in the presence of acetone medium. Various samples of PANI- $\mathrm{CoFe}_{2} \mathrm{O}_{4}$ composite with varying amount of ferrite $(5,10,15 \mathrm{~g})$ in $0.4 \mathrm{M}$ PANI synthesized are designated as $\mathrm{P} 4 \mathrm{C} 5, \mathrm{P} 4 \mathrm{C} 10$ and $\mathrm{P} 4 \mathrm{C} 15$ respectively.

The FTIR spectra was recorded on a ThermoNicolate Avatar370 (Model) spectrophotometer in $\mathrm{KBr}$ medium in the region 4000-400 $\mathrm{cm}^{-1}$ having DTGS detector. The phase analysis of the samples was carried out using Rigaku D max-B model $\mathrm{X}$-ray diffractometer using $\mathrm{Cu} \mathrm{K} \alpha$ radiations. SEM analysis of the samples is done with the SEM make JEOL Model JSM-6390LV. Room temperature magnetic measurements of the ferrite and PANI composites are carried out by VSM: model Lakeshore VSM 7410. Dielectric properties of the samples are measured using precision impedance analyzer Wayen Kerr 6500B in the frequency range of $100 \mathrm{~Hz}$ to $20 \mathrm{MHz}$.

\section{Results and Discussions:-}

\section{XRD}

X-ray diffractogram of PANI, CF and P4C5 composite is depicted in Figure1. The characteristic peaks of PANI observed at $2 \theta$ values $25^{\circ}$ and at $20^{\circ}$ ensure the formation of PANI in the conductive emeraldine base form. The XRD pattern of cobalt ferrite matches with the JCPDS no. 22-1086. Characteristic peaks of cobalt ferrite are observed in the XRD pattern of P4C5. There is no shift in peak position of $\mathrm{CF}$ in composite which indicates that ferrite retained its structure even though it is dispersed in PANI during the polymerization reaction. The average particle size was determined by the Debye Scherrer equation

$$
t=\frac{0.9 \lambda}{\beta \cos \theta}
$$

where ' $\mathrm{t}$ ' is the thickness of the crystallite (particle size), $\lambda$ is the wavelength of $\mathrm{Cu} \mathrm{K \alpha}$ $\left(1.5406 \mathrm{~A}^{0}\right), \beta$ is the angular width which is equal to the full width half maximum. The calculated particle size of ferrite and the composite are 11.89 and $13.4 \mathrm{~nm}$ respectively. The small increase in size may due to the coating of ferrite particles with PANI.

\section{IR}

The IR spectra of CF, PANI and P4C5 are shown in Figure 2. The bands at 1569, 1485, 1292, 1111 and $798 \mathrm{~cm}^{-1}$ corresponds to polyaniline. Absorption band at $798 \mathrm{~cm}^{-1}$ is due to out of plane bending vibration of C-H bond of 
para substituted benzene ring which confirms the conjugated $\pi$ system present in PANI. The bands corresponding to stretching vibrations of $\mathrm{N}-\mathrm{B}-\mathrm{N}$ (benzenoid) and $\mathrm{N}=\mathrm{Q}=\mathrm{N}$ (qunanoid) structures appear at 1485 and $1569 \mathrm{~cm}^{-1}$ respectively confirms the formation PANI chain in the most conductive emeraldine form. A strong band appears at $1110 \mathrm{~cm}^{-1}$ has been explained as an in plane bending vibration of imino-1,4-phenylene and has been reported to be associated with the electrical conductivity of PANI.

All the characteristic peaks of PANI are observed in the IR spectrum of composite, showing that the introduction of ferrite nanoparticles does not damage the backbone structure of PANI. The peak at around $503 \mathrm{~cm}^{-1}$ in the composite confirms the presence of ferrite particles in the composite.

\section{Morphology studies}

SEM images of CF, PANI and P4C5 composite are depicted in the Figure 3. Monodispersed spherical particles are observed in the SEM image of CF. Nano magnetic particles are easily agglomerated, which can also observed in the SEM image of CF. Morphology of PANI is changed due to the incorporation of CF nano particles.Uniform distribution of spherical shaped particle is observed in the SEM of PANI. In the composite spherical shaped particles are retained with more uniform distribution.

\section{Magnetic Studies}

The hysteresis loops for PANI, CF and PANI-ferrite composites are measured at room temperature and are shown in Figure 4, 5a and 5b. Parameters like saturation magnetization (Ms), coercivity (He) and magnetic remanance(Mr) are tabulated in table 1 .

Table 1:-Magnetic Characteristics of PANI, CF and Composites

\begin{tabular}{|l|l|l|l|}
\hline Sample & $\begin{array}{l}\text { Saturation } \\
\text { MagnetisationMs }(\mathbf{e m u} / \mathbf{g})\end{array}$ & CoercivityHc (Oe) & Magnetic RemananceMr(emu/g) \\
\hline PANI & 0.056 & 231 & 0.01 \\
\hline CF & 53.37 & 1411 & 16.38 \\
\hline P4C5 & 27.72 & 1502 & 9.02 \\
\hline P4C10 & 31.37 & 1483 & 10.39 \\
\hline P4C15 & 35.61 & 1516 & 11.39 \\
\hline
\end{tabular}

Saturation magnetization of cobalt ferrite is 53.37. Msvalues of the composites is lower than that of CF as it is coated with PANI and is linearly dependant on the amount of ferrite. Coercivity of the composites is found to be higher than that of pure CF. As remanance magnetization is quantitatively depends on the amount of ferrite, it is found to increase with the amount of ferrite in the composites.

\section{Dielectric studies}

The variation of the dielectric constant $\varepsilon^{\prime}$ of $\mathrm{CF}$, PANI and composites as a function of logarithmic frequency is plotted in Figure 6-8. In all the samples, dielectric constant decreases with increase in frequency. This dependence upon frequency is because of the interfacial polarization as predicted by Maxwell-Wagner. According to this model, the dielectric structure of a ferrite material is assumed to be made up of two layers; the first layer being a conductive layer that consists of large ferrite grains and the other being the grain boundaries that are poor conductors. The high value of dielectric permittivity at lower frequency can be accounted by employing Koop's theory which is based on Maxwell-Wagner model for heterogeneous double layer. Under the application of an external ac electrical field, an electron exchange between +2 and +3 oxidation state occurs, resulting in local displacement of charge in the direction of the applied field. This electron exchange determines the net polarization effect in ferrites. At lower applied frequency the hopping frequency of electron between +2 and +3 state will be high and polarization is maximum. Hence the sample gives high permittivity. As the frequency increases, hopping frequency lags behind the applied frequency, thus dielectric polarization as well as the dielectric permittivity decreases.

The dielectric permitivity of PANI is in the range of $10^{4}$ and that of ferrites and composites are in the range of $10^{3}$.The dielectric properties of PANI and composites are very large at lower frequencies and found to be decrease steeply with increase in frequency.Variation of dielectric permittivity with respect to the amount of ferrite in composites at different frequency is plotted in figure 9. The dielectric permitivity of PANI decreases with the incorporation of ferrites. The permitivity value of composites increases with the ferrite content. Maximum value is obtained for P4C10. 


\section{Ac conductivity}

Variation of ac conductivity of CF, PANI and composites are plotted in figure 10-12. The ac conductivity initially increases with frequency, reaches a maximum and then decreases at higher frequencies. This can be explained using Maxwell-Wagner two layers model structure of ferrites, in which the conducting grains are separated by nonconducting grain boundaries. At low frequencies resistive grain boundaries are active whereas at higher frequencies conducting grains became active. PANI exhibits ac conductivity higher than that of cobalt ferrite. Ac conductivity of all the three composites is found to be lower than that of pure PANI. The decrease is due to the distribution of ferrite particles in PANI chain that may affect the conduction. In the three series of composites an increase in conductivity is observed with loading of ferrite from $5 \mathrm{~g}$ to $10 \mathrm{~g}$. Further increase in the loading to $15 \mathrm{~g}$ decreases the conductivity which indicates that high concentration of ferrite particles cannot incorporate in the PANI matrix.

\section{Conclusions:-}

Green chemistry is an area of chemistry and chemical engineering focused on the designing of products and processes that minimize the use and generation of hazardous substances. It is reported that the corrosive nature of the mineral acids may affect the properties of the filler inorganic nano particle in the polymer matrix of composites.In this work, PANI-CoFe ${ }_{2} \mathrm{O}_{4}$ nano composites have been successfully synthesized via insitu polymerization of aniline in presence of $\mathrm{CoFe}_{2} \mathrm{O}_{4}$ nano particles in green solvent tamarind extract. It is focused on technological approaches to prevent pollution and reducing consumption of nonrenewable resources. The use of safe, environmental friendly solvent is one of the important principles of green chemistry. The present study aimed and revealed that tamarind extract, a naturally occurring product, is an effective green solvent for the synthesis of polyaniline nanocomposites having both electric and magnetic properties. It is also found that the electric and magnetic properties can be tuned by varying the amount of ferrite particles.

\section{Acknowledgements:-}

We thank Department of Physics, Maharajas College, Ernakulam, Department of Physics, S.D.College, Alappuzha, SAIF, IIT, Madras and SAIF, CUSAT, Cochin, for the analysis.

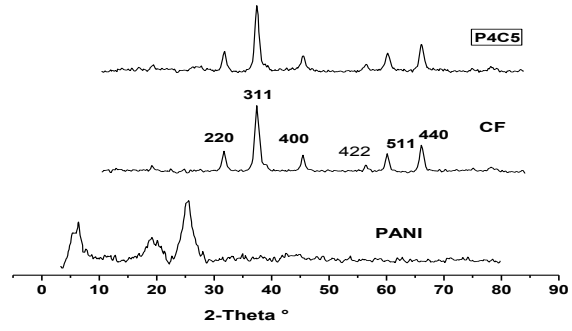

Figure 1:-X- ray diffractogram of PANI, CF and P4C5

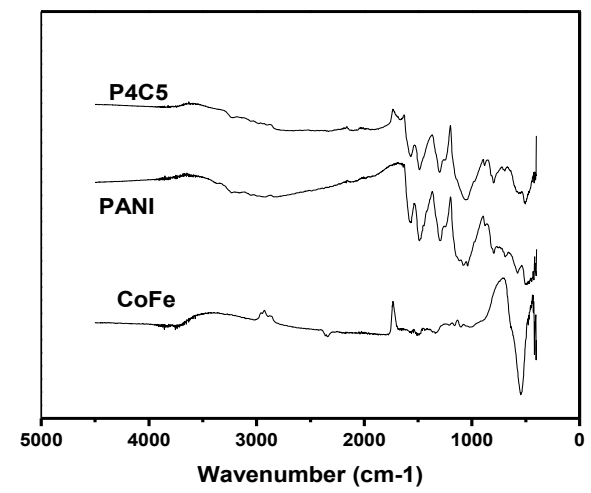

Figure 2:-IR spectra of CF, PANI and P4C5 

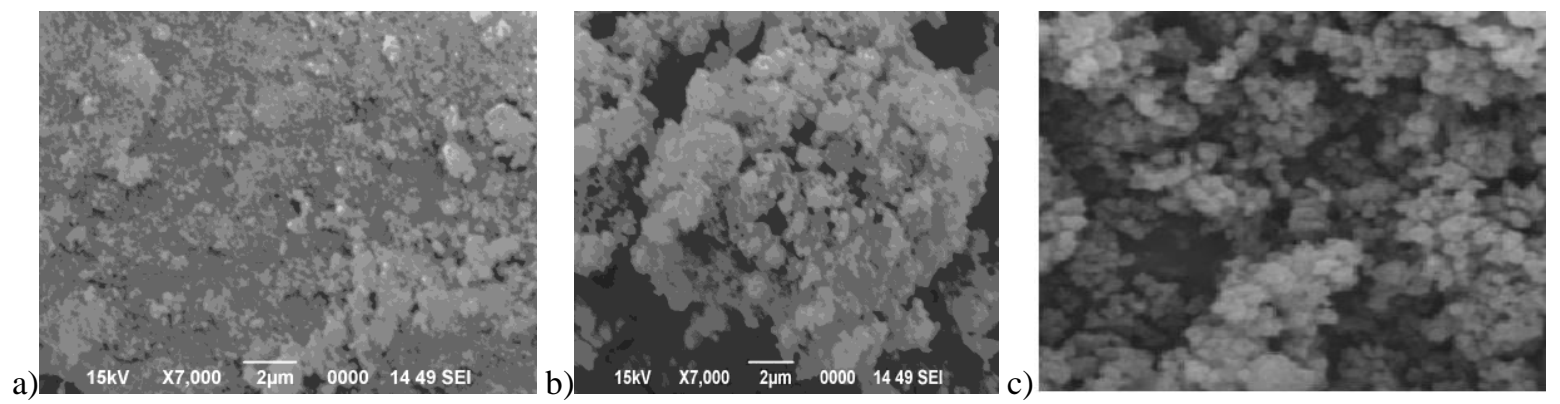

Figure 3:-SEM images of a) CF, b) PANI and c) P4C5 composite

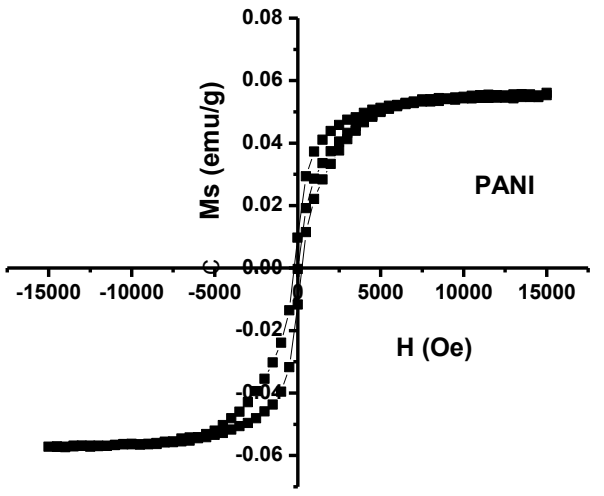

Figure 4:-Hysteresis loop for PANI

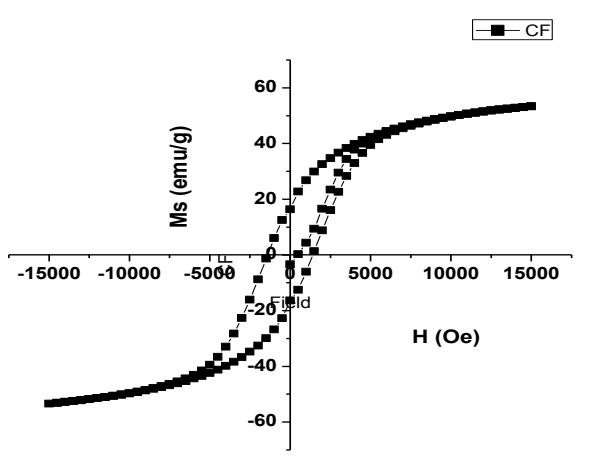

Figure 5:-a. Hysteresis loop for CF 


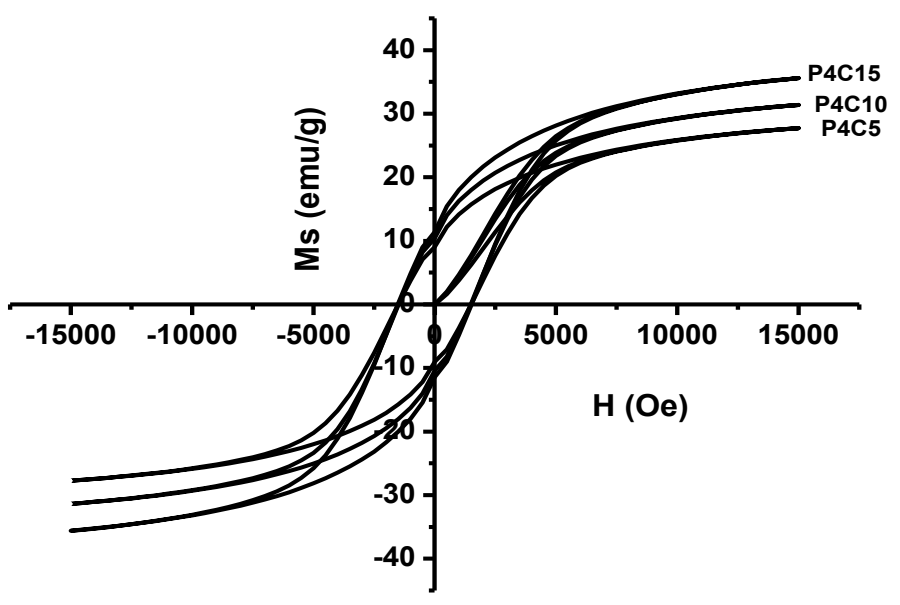

Figure 5:-b. Hysteresis loop for PANI-ferrite composites

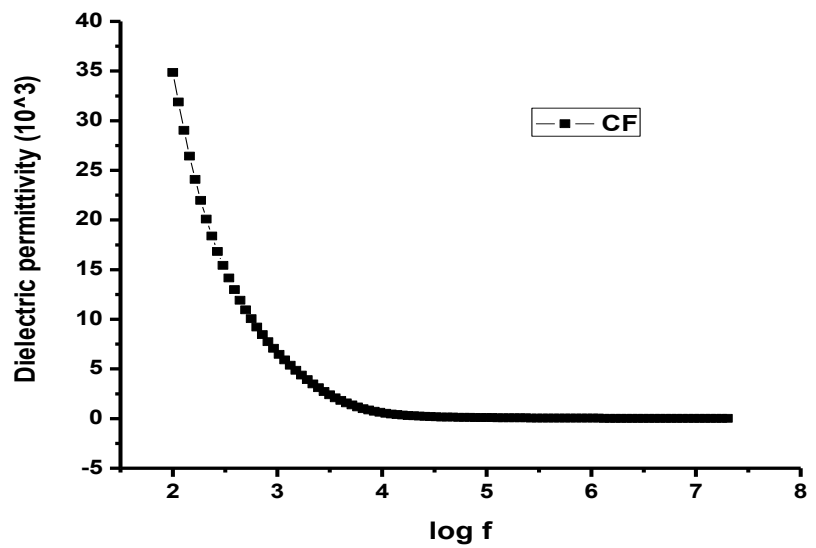

Figure 6:-Frequency dependence of dielectric permittivity of cobalt ferrite

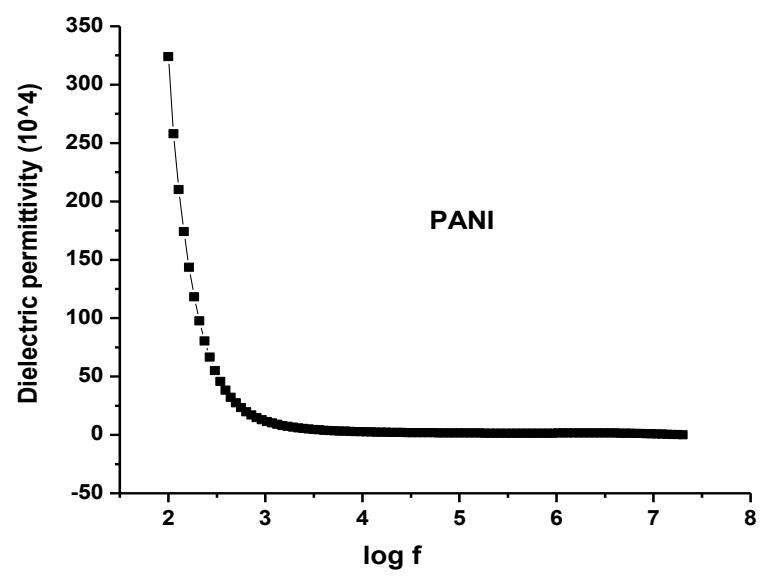

Figure 7:-Frequency dependence of dielectric permittivity of PANI 


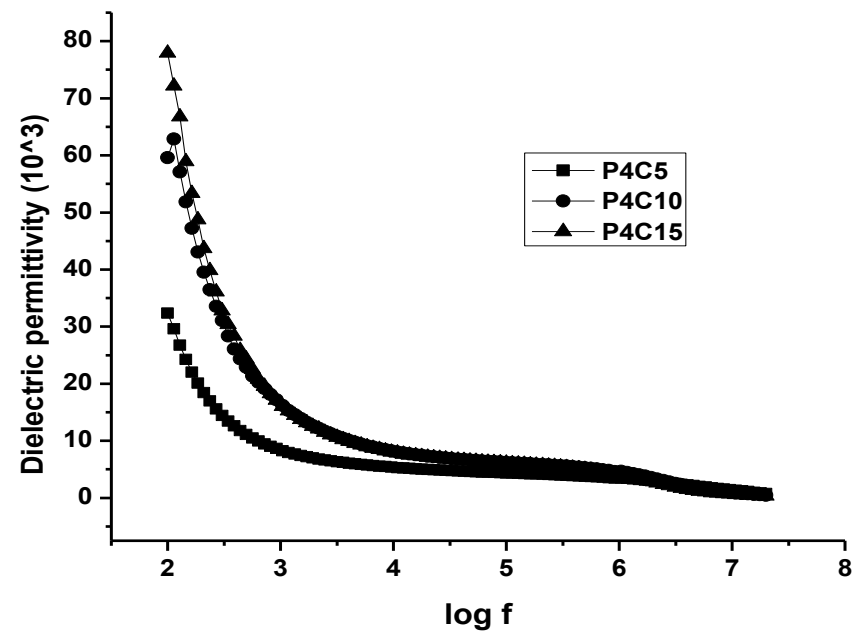

Figure 8:-Variation of dielectric constant of the composites with frequency

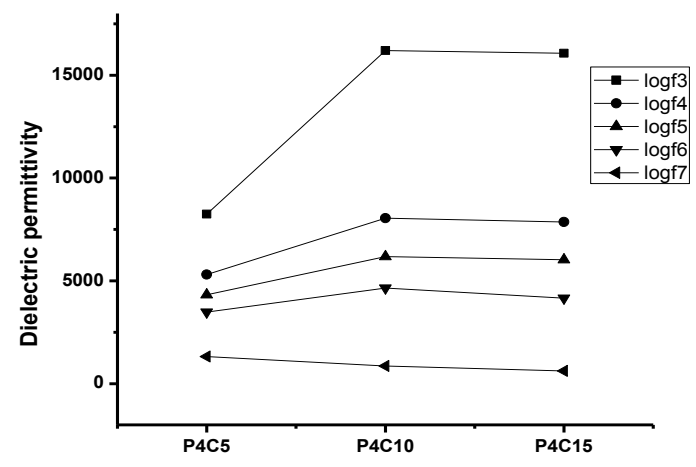

Figure 9:-Loading dependence of dielectric permittivity

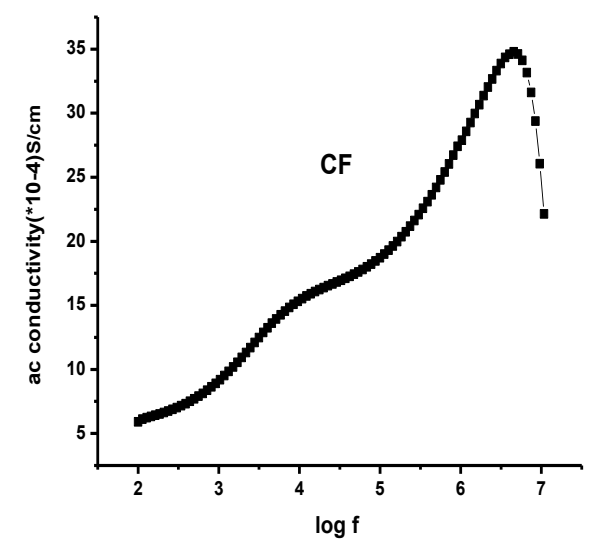

Figure 10:-Frequency dependence of ac conductivity of cobalt ferrite 


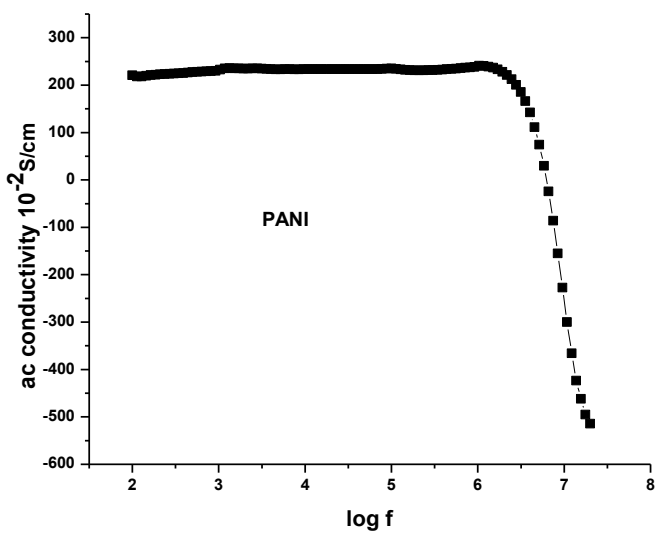

Figure 11:-Frequency dependence of ac conductivity of PANI

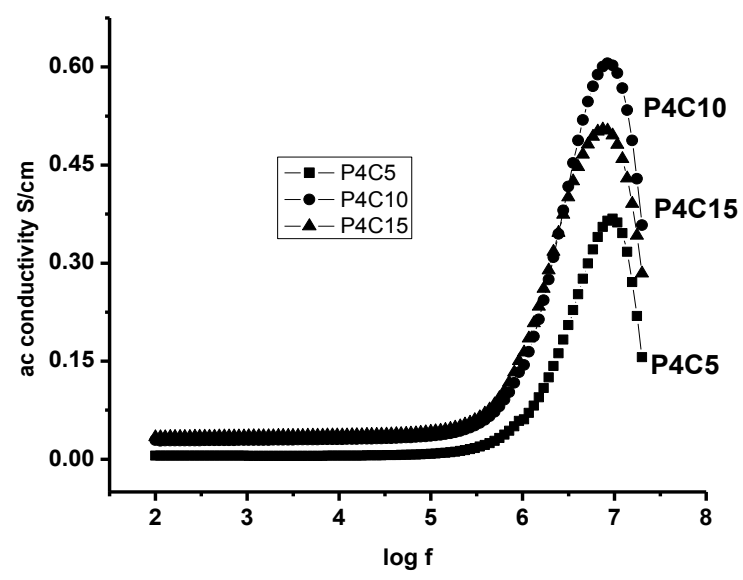

Figure 12:-Variation of ac conductivity of the composites with frequency

\section{References:-}

1. Jiaxing Huang, (2006) Syntheses and applications of conducting polymer polyaniline nanofibers, J.Pure Appl. Chem., 78, No. 1, 15-27.

2. Prasanna G.D., Ashok R.L, Prasad V.B. and Jayanna H.S, (2014)Synthesis and characterization of magnetic and conductive nickel ferrite-polyaniline nanocomposites, Journal of Composite Materials, $0(0), 1-9$.

3. Stejskal J. and Gilbert R. G., (2002) Polyaniline. Preparation of a Conducting Polymer (IUPAC Technical Report), Pure and Applied Chemistry, 74, No. 5, 857-867.

4. Subhash B. Kondawar, Arti I.Nandapure and Bharti I.Nandapure (2014) Nanocrystalline nickel ferrite reinforced conducting polyaniline nanocomposites, J.Adv.Mat.Lett., 5(6), 339-344.

5. Tan Y., Zhang Y. and Kan J. (2009) Synthesis and properties on polyaniline in the presence of nickel chloride, J. eXPRESS Polymer Letters, Vol.3, No.6,333-339.

6. Tanriverdi E.E., Uzumcu A.T., Kavas H.,Demir A. and Baykal A., (2011) Conductivity Study of PolyanilineCobalt Ferrite (PANI-CoFe2O4) Nanocomposite, J.Nano-Micro Lett. 3 (2), 99-107.

7. Tiwari A., Singh V.,( 2007) Synthesis and characterization of electrical conducting chitosan-graft-polyaniline, J.eXPRESS Polymer Letters, Vol.1, No.5,308-317

8. Veluru Jagadeesh Babu, Sesha Vempati, Seeram Ramakrishna, (2013) Conducting Polyaniline-Electrical Charge Transportation, J.Materials Sciences and Applications, 4, 1-10.

9. Yongli Li, Chunxia Zhu and Jinqing Kan, (2015) Preparation and Characteristics of $\gamma$-Fe2O3/PolyanilineCurcumin Composites,J.Metals, 5, 2401-2412. 